\title{
Discharge by Noon: Toward a Better Understanding of Benefits and Costs
}

\author{
Aline Zorian, MD¹, Dan Shine, MD², Michelle Mourad, MD* \\ ${ }^{1}$ Division of Hospital Medicine, University of California San Francisco, San Francisco, California; ${ }^{2}$ Department of Medicine, University of Colorado \\ School of Medicine, Aurora, Colorado.
}

ras

argeting "discharge before noon" (DBN) for hospitalized patients has been proposed as a way to improve hospital throughput and patient safety by reducing emergency department (ED) boarding and crowding. In this issue, Kirubarajan et al ${ }^{1}$ report no association between morning discharge and length of stay (LOS) for either the ED or hospitalization. ${ }^{1}$ This large (189,781 patients) 7-year study from seven quite different Canadian hospitals adds important data to a literature that remains divided about whether DBN helps or hurts hospital LOS and ED boarding.

Unlike trials reporting interventions to encourage DBN, this observational study was unique in that it took each day as the unit of observation. This method cleverly allowed the authors to examine whether days with more discharges before noon conferred a lower mean ED and inpatient LOS among patients admitted on those days. Their approach appropriately reframes the central issue as one of patient flow.

Kirubarajan et al's most notable, and perhaps surprising, finding is the lack of association between morning discharge and ED LOS. Computer modeling supports the hypothesis that ED throughput will improve on days with earlier inpatient bed availability. ${ }^{2}$ Several studies have also noted earlier ED departure times and decreased ED wait times after implementing interventions to promote DBN. ${ }^{3}$ Why might the authors' findings contradict previous studies? Their outcomes may in part be due to high ED LOS (>14 hours), exceeding Canadian published targets and reports from the United States. ${ }^{4,5}$ Problems relating to ED resources, practice, and hospital census may have overwhelmed DBN as factors in boarding. The interpretation of their findings is limited by the authors' decision to report only ED LOS, rather than including the time between a decision to admit and ED departure (boarding time).

While early studies that focused on interventions to promote DBN noted decreased inpatient LOS after their implementation, later studies found no effect or even an increase in LOS for general internal medicine patients. Concerns have been raised about the confounding effect of concurrent initiatives aimed at improving LOS as well as misaligned incentives to delay discharge to the following morning. As the number of conflicting studies mounts, and with the current

*Corresponding Author: Michelle Mourad, MD; Email: Michelle.mourad@ ucsf.edu; Telephone: 415-476-2264; Twitter: @Michelle_Mourad.

Received: February 18, 2021; Revised: March 1, 2021; Accepted: March 1, 2021

(c) 2021 Society of Hospital Medicine DOI 10.12788/jhm.3613 report in hand, it is tempting to conclude that for the DBN evidence base as a whole, we are observing random variation around no effect.

With growing doubt about benefits of morning discharge, perhaps we should turn our attention away from the question of how to increase DBN and consider instead why and at what cost. Hospitals are delicate organisms; a singular focus on one metric will undoubtedly impact others. Does the effort to discharge before noon consume valuable morning hours and detract from the care of other patients? Are patients held overnight unnecessarily to comply with DBN? Are there consequences in patient, nursing, or trainee satisfaction? Is bedside teaching affected?

And as concepts of patient-centered care are increasingly valued, we may ask whether DBN is such a concept, or is it rather an increasingly dubious strategy aimed at regularizing hospital operations? The need for a more holistic assessment of "discharge quality" is apparent. Instead of focusing on a particular hour, initiatives should determine the "best, earliest discharge time" for each patient and align multidisciplinary efforts toward this patient-centered goal. Such efforts are already underway in pediatric hospitals, where fixed discharge times are being replaced by discharge milestones embedded into the electronic medical record. ${ }^{6}$ An instrument to track "discharge readiness" such as this one, paired with ongoing analysis of the barriers to timely discharge, might better facilitate throughput by targeting the entire admission, rather than concentrating pressure on its final hours.

Disclosures: The authors have no conflicts to disclose.

\section{References}

1. Kirubarajan A, Shin S, Fralick M, Kwan Jet al. Morning discharges and patient length-of-stay in inpatient general internal medicine. J Hosp Med. 2021;16(6):334-338. https://doi.org/ 10.12788/jhm.3605

2. Powell ES, Khare RK, Venkatesh AK, Van Roo BD, Adams JG, Reinhardt G. The relationship between inpatient discharge timing and emergency department boarding. J Emerg Med. 2012;42(2):186-196. https://doi.org/10.1016/j. jemermed.2010.06.028

3. Wertheimer B, Jacobs RE, Iturrate E, Bailey M, Hochman K. Discharge before noon: effect on throughput and sustainability. J Hosp Med. 2015;10(10):664669. https://doi.org/10.1002/jhm.2412

4. Fee C, Burstin H, Maselli JH, Hsia RY. Association of emergency department length of stay with safety-net status. JAMA. 2012;307(5):476-482. https://doi. org/10.1001/jama.2012.41

5. Ontario wait times. Ontario Ministry of Health and Ministry of Long-Term Care. Accessed February 17, 2021. http://www.health.gov.on.ca/en/pro/programs/waittimes/edrs/targets.aspx

6. White $C M$, Statile $A M$, White $D L$, et al. Using quality improvement to optimise paediatric discharge efficiency. BMJ Qual Saf. 2014;23(5):428-436. https://doi.org/10.1136/bmjqs-2013-002556 